\title{
Reaction Extent and Mass Conservation(s)
}

Diego J. Raposo ${ }^{1 *}$

\begin{abstract} conservation (between different substances in a chemical reaction) in closed systems.

Keywords

Reaction extent - Stoichiometry - Mass conservation - Closed systems

${ }^{1}$ Departamento de Química Fundamental, Universidade Federal de Pernambuco, Pernambuco, Brazil

*Corresponding author: diego.rsilva@ufpe.br
\end{abstract}

A new proof to introduce reaction extent in General Chemistry courses, with mathematical and chemical arguments, is suggested, together with the relation between mass conservation (over time) and reaction extent

\section{Contents}

\section{Introduction}

1 Reaction extent demonstration

2 Mass vs. reaction extent conservations

3 Mass conservation in chemical reactions

4 Conclusions

Acknowledgments

References

\section{Introduction}

Reaction extent is a well-defined quantity that relates all components of a system that take part in a chemical reaction. It was firstly defined by Théophile De Donder [1] and, since then, have permeated many applications [2] in conceptual teaching of Equilibrium Thermodynamics $[3,4]$ and Kinetics [5, 6], and also in the understanding of complex processes occurring outside equilibrium $[7,8]$. There are very good reasons to teach the concept during Stoichiometry lessons for General Chemistry courses [9], and according to Moretti, "to use the concept of extent of reaction may be the first simple way of attaining a mathematization of chemistry, which is very important for the advance of chemistry" [10].

The problem is that reaction extend is usually introduced and then subsequently applied (in Physical Chemistry books), without a proper contemplation of an important property it has: its conservation within closed systems. Without it we wouldn't be able to estimate the rate of a chemical reaction by following the evolution of the amount of any reagent or product over time (assuming the stoichiometry of the reaction is well known) [9], for instance. This conservation simply means that the reaction extent can be calculated from any reagent or product at a given time in a specific chemical reaction in the system, because it is a characteristic quantity of the reaction, and not from specific reacting components in the phase [2]. More importantly, this "conservation" can be used to demonstrate the presence of a "constant" through chemical changes in a given time interval, which is the reaction extent. Now this quantity can be derived rather than defined from the beginning.

Another approach, used mostly in Thermochemistry books, is the definition of reaction extent as a consequence of the law of definite proportions [11, 12] (which was, by the way, the original approach of De Donder). 5 This law is also used in our demonstration, but not from start, and not in its usual form. We rather make a logical construction from basic principles that characterize the chemical reaction, and the law appears as a reasonable assumption in the end. Moreover, the aforementioned approach also does not emphasize reaction extent conservation, as we plan to do.

Therefore, in Section 1 we present an alternative demonstration for the reaction extent presence in a reaction, without assuming the law of definite proportions explicitly, and using the search for a constant (or conserved) quantity over chemical changes as a guide through the proof. In this demonstration, the interpretation of reaction extent as a "reaction counter" (quantifying the number of chemical processes after some time interval) and the importance of the precise knowledge of the reaction stoichiometry to define it are highlighted.

The use of the term "conservation", if not addressed properly, may have a drawback: the confusion between mass conservation and reaction extent conservation. We dedicate sections 2 (mass conservation) and 3 (reaction extent conservation) to clarify the matter, but the main idea is: the first is a conservation over time, and the second is a conservation over chemical changes (changes in the amount of any species in the reaction) in a given time interval. In mathematical terms, we define conservation of a quantity $y$ with relation to a quantity $x$ if $d y / d x$ is a constant (a function which is independent on both $x$ and $y$ ). In mass conservation $y$ is mass and $x$ is time; so 
the conservation is such that $\mathrm{dy}(\mathrm{x}) / \mathrm{d} x=$ constant $=0$. In reaction extent conservation, $y$ is the reaction extent, and $x$ is the amount of any component which is present in the chemical reaction. Both quantities depend on time, but given a time interval we can show that $\mathrm{d} y(x) / \mathrm{d} x=$ constant; this constant is time independent, different from zero (in contrast with mass conservation) and depends on the stoichiometry of the reaction, only.

The "reaction extent demonstration" presented in this paper (Section 1) is suitable for General Chemistry courses, as a way of introduce the quantity previous to its applications in Stoichiometry [9, 13], and limiting reagent concepts [14]. Then the students would benefit greatly on more advanced steps, when Chemical Kinetics and Thermodynamics are introduced in correlation with the reaction extent. The precise meaning of conservation of the reaction extent and its relation with mass conservation (Sections 2 and 3) may have advantages in the initial steps of Chemical Kinetics classes, or in Thermodynamics as taught in Chemistry and Engineering courses.

\section{Reaction extent demonstration}

Consider a reaction $3 \mathrm{~A} \longrightarrow 2 \mathrm{~B}$. If 3 molecules of $\mathrm{A}$ reacts, and there were initially 3 of them, in the end none remain, so the molecular variation - the difference between the final and the initial number of molecules, $N_{f}$ and $N_{i}$, respectively - is $\Delta N=N_{f}-N_{i}=0-3=-3$. That leads, according the reaction equation, to 2 molecules of B where there were none: $\Delta N^{\prime}=N_{f}^{\prime}-N_{i}^{\prime}=2-0=2$. Obviously, the mass of the system hasn't changed, but the number of each molecule did. So how could we scribe a quantity that says to us the reaction just occurred, in terms of the molecules that had to react and in fact did? We know the variations $\Delta \mathrm{N}$ and $\Delta \mathrm{N}^{\prime}$ are connected, since they pertain to the same reaction: $\Delta N \leftrightarrow \Delta N^{\prime}$. But if one defines a quantity that is conserved through variations of one substance compared to variations in any other, that would be chemically very useful, since to deal with amounts of individual species compared to others in a reaction is the main goal of Stoichiometry. The way of doing that is to turn $\Delta N \leftrightarrow \Delta N^{\prime}$ into an equality, by dividing the molecular variation by proper numbers:

$$
\begin{gathered}
\Delta \mathrm{N}=0-3 \leftrightarrow 2-0=\Delta \mathrm{N}^{\prime} \\
\frac{\Delta \mathrm{N}}{-3}=\frac{0-3}{-3}=\frac{2-0}{2}=\frac{\Delta \mathrm{N}^{\prime}}{2}=1
\end{gathered}
$$

Observe that dividing the molecular variation by the substance's stoichiometric number (the negative of the stoichiometric coefficient for reagents, and the stoichiometric coefficient for products) the association becomes equality, and this normalization leads to a number: 1 . This number tells that one reaction (or "reaction event"
[9]), as defined, occurred. It is not hard to generalize this for a prototype reaction:

$$
\mathrm{a}_{1} \mathrm{~A}_{1}+\mathrm{a}_{2} \mathrm{~A}_{2}+\ldots \longrightarrow \mathrm{b}_{1} \mathrm{~B}_{1}+\mathrm{b}_{2} \mathrm{~B}_{2}+\ldots
$$

Therefore, in order to 1 reaction event occurs, the changes in molecules number are such that:

$$
\begin{aligned}
\Delta \mathrm{N}_{\mathrm{A}_{1}} & =0-\mathrm{a}_{1} \\
\Delta \mathrm{N}_{\mathrm{A}_{2}} & =0-\mathrm{a}_{2} \\
& \ldots \\
\Delta \mathrm{N}_{\mathrm{B}_{1}} & =\mathrm{b}_{1}-0 \\
\Delta \mathrm{N}_{\mathrm{B}_{2}} & =\mathrm{b}_{2}-0 \\
\ldots &
\end{aligned}
$$

And, obviously, to associate the variations in a single quantity, which is " 1 reaction" or " $1 \mathrm{rxn}$ " as suggested previously [13]:

$$
\begin{gathered}
\Delta \mathrm{N}_{\mathrm{A}_{1}} \leftrightarrow \Delta \mathrm{N}_{\mathrm{A}_{2}} \leftrightarrow \ldots \leftrightarrow \Delta \mathrm{N}_{\mathrm{B}_{1}} \leftrightarrow \Delta \mathrm{N}_{\mathrm{B}_{2}} \leftrightarrow \ldots \\
0-\mathrm{a}_{1} \leftrightarrow 0-\mathrm{a}_{2} \leftrightarrow \ldots \leftrightarrow \mathrm{b}_{1}-0 \leftrightarrow \mathrm{b}_{2}-0 \leftrightarrow \ldots \\
\frac{0-\mathrm{a}_{1}}{-\mathrm{a}_{1}}=\frac{0-\mathrm{a}_{2}}{-\mathrm{a}_{2}}=\ldots=\frac{\mathrm{b}_{1}-0}{\mathrm{~b}_{1}}=\frac{\mathrm{b}_{2}-0}{\mathrm{~b}_{2}}=\ldots=1 \mathrm{rxn} \\
\frac{\Delta \mathrm{N}_{\mathrm{k}}}{v_{\mathrm{k}}}=1 \mathrm{rxn}(\forall \mathrm{k})
\end{gathered}
$$

Where " $\forall k$ " is a shorthand notation of "for all species $k^{\prime \prime}$, and the stoichiometric number for a substance $k$ is defined as:

$$
v_{k}:= \begin{cases}-a_{i} & \text { for a reagent } A_{i} \\ b_{j} & \text { for a product } B_{j}\end{cases}
$$

The symbol " $:=$ " means that the term on the left of the equation is defined as the term on the right.

Therefore, once the quantity defined "tells" when one reaction occurs, let's see when more reactions are taking place. Once again using the previous example, $3 \mathrm{~A} \longrightarrow 2 \mathrm{~B}$, if we have 3 mols of $\mathrm{A}$ generating 2 mols of $\mathrm{B}$, then $\Delta \mathbf{N}=0-3 \mathrm{~mol} \leftrightarrow 2-0 \mathrm{~mol}=\Delta \mathbf{N}^{\prime}$, with $\Delta \mathbf{N}$ meaning the change in $\mathrm{A}^{\prime} \mathrm{s}$ quantity in order to $1 \mathrm{~mol}$ of reactions (just the Avogadro's number of reactions) occur is $-3 \mathrm{~mol}$, leading to a increase of $2 \mathrm{~mol}$ of $\mathrm{B}$ $\left(\Delta \mathbf{N}^{\prime}=2 \mathrm{~mol}\right)$. Making it general:

$$
\begin{gathered}
\Delta \mathbf{N}_{\mathrm{A}_{1}} \leftrightarrow \ldots \leftrightarrow \Delta \mathbf{N}_{\mathrm{B}_{1}} \leftrightarrow \ldots \\
\left(0-\mathrm{a}_{1}\right) \mathrm{mol} \leftrightarrow \ldots \leftrightarrow\left(\mathrm{b}_{1}-0\right) \mathrm{mol} \leftrightarrow \ldots \\
\frac{\left(0-\mathrm{a}_{1}\right) \mathrm{mol}}{-\mathrm{a}_{1}}=\ldots=\frac{\left(\mathrm{b}_{1}-0\right) \mathrm{mol}}{\mathrm{b}_{1}}=\ldots=1 \mathrm{~mol}-\mathrm{rxn} \\
\frac{\Delta \mathbf{N}_{\mathrm{k}}}{v_{\mathrm{k}}}=1 \mathrm{~mol}-\operatorname{rxn}(\forall \mathrm{k})
\end{gathered}
$$


What relates the variation in molecular numbers or in substance quantities through equality is the normalization by stoichiometric numbers, since they lead to the same result for each substance: either 1(rxn) or 1 mol(rxn). Naturally the number of reactions needs not to be an integer, or an integer multiple of $6.02 \times 10^{23}$. Since the amount of substance $A_{1}$ that changes have to be a multiple of $a_{1}$, the proportionality constant, $\xi$, is such that $n_{A_{1}}=\xi a_{1}$ mol (this is statement of law of definite proportions, if the relation between the amount of substance with its mass is used). Doing that for variations in any substance quantities, $\Delta \mathrm{n}$ :

$$
\begin{gathered}
\Delta \mathrm{n}_{\mathrm{A}_{1}} \leftrightarrow \ldots \leftrightarrow \Delta \mathrm{n}_{\mathrm{B}_{1}} \leftrightarrow \ldots \\
\left(0-\xi \mathrm{a}_{1}\right) \mathrm{mol} \leftrightarrow \ldots \leftrightarrow\left(\xi \mathrm{b}_{1}-0\right) \mathrm{mol} \leftrightarrow \ldots \\
\frac{\left(0-\xi \mathrm{a}_{1}\right) \mathrm{mol}}{-\mathrm{a}_{1}}=\ldots=\frac{\left(\xi \mathrm{b}_{1}-0\right) \mathrm{mol}}{\mathrm{b}_{1}}=\ldots=\xi \mathrm{mol}-\mathrm{rxn} \\
\frac{\Delta \mathrm{n}_{\mathrm{k}}}{v_{\mathrm{k}}}=\xi \mathrm{mol}-\mathrm{rxn}(\forall \mathrm{k})
\end{gathered}
$$

To that quantity - which is the amount of reaction performed [13], and that can be found by variations in particle's (or mols) number of any species involved - it was given the name reaction extent. The reaction extent is a reaction-counter, and since its definition its meaning was clear: "how many complete reactions have taken place" [15]. Since $n_{k}$ is a quantity that changes through time, as much as $\xi$, both can be differentiated and integrated, according to proper boundaries in time:

$$
\begin{gathered}
\frac{\mathrm{dn}_{\mathrm{k}}(\mathrm{t})}{v_{\mathrm{k}}}=\mathrm{d} \xi(\mathrm{t})(\forall \mathrm{k}) \\
\frac{1}{v_{k}} \int_{n_{k}\left(\mathrm{t}_{0}\right)}^{n_{k}(t)} \mathrm{dn}_{\mathrm{k}}\left(\mathrm{t}^{\prime}\right)=\int_{\xi\left(\mathrm{t}_{0}\right)}^{\xi(t)} \mathrm{d} \xi\left(\mathrm{t}^{\prime}\right)(\forall \mathrm{k}) \\
\frac{\mathrm{n}_{\mathrm{k}}(\mathrm{t})-\mathrm{n}_{\mathrm{k}}\left(\mathrm{t}_{0}\right)}{v_{\mathrm{k}}}=\xi(\mathrm{t})-\xi\left(\mathrm{t}_{0}\right)(\forall \mathrm{k})
\end{gathered}
$$

In the beginning of a reaction we set the time $t_{0}=$ 0 , and define $\xi\left(t_{0}\right):=0[3,12]$, since no reaction have occurred:

$$
\xi(t)=\frac{n_{k}(t)-n_{k, 0}}{v_{k}}(\forall k)
$$

Where $n_{k, 0}=n_{k}\left(t_{0}=0\right)$ is the initial amount of $k$ (which is time independent). If we differentiate both sides of Eq. 6 with respect to time (and omit the time dependence of $n$ and $\xi$ to simplify the notation):

$$
\frac{\mathrm{d} \xi}{\mathrm{dt}}=\frac{1}{v_{\mathrm{k}}} \frac{\mathrm{d} n_{\mathrm{k}}}{\mathrm{dt}}(\forall \mathrm{k})
$$

In another form, by comparing two substances $x$ and $y$, that share the same reaction extent change:

$$
\frac{1}{v_{x}} \frac{\mathrm{d} n_{x}}{d t}=\frac{1}{v_{y}} \frac{d n_{y}}{d t}(\forall x, y)
$$

Here we found an expression that relates variations in quantities of different species.

\section{Mass vs. reaction extent conservations}

If a chemical reaction is performed in a closed system the total mass is conserved, and the sum of initial masses of all reagents $\left(m_{R, 0}\right)$ and all products $\left(m_{P, 0}\right)$ is equal to the sum of the final masses of both at time $t$ :

$$
\begin{gathered}
m_{R, 0}+m_{P, 0}=m_{R, t}+m_{P, t} \\
m_{R, t}-m_{R, 0}=m_{P, 0}-m_{P, t} \\
m_{R, t}-m_{R, 0}=-\left(m_{P, t}-m_{P, 0}\right)
\end{gathered}
$$

Defining mass variations $\Delta \mathrm{m}$ as differences between final and initial states:

$$
\Delta \mathrm{m}_{\mathrm{R}}=-\Delta \mathrm{m}_{\mathrm{P}}
$$

Logically, if this variation is over time, then:

$$
\frac{\Delta \mathrm{m}_{\mathrm{R}}}{\Delta \mathrm{t}}=-\frac{\Delta \mathrm{m}_{\mathrm{P}}}{\Delta \mathrm{t}}
$$

For infinitesimal changes, Eq. 11 leads to:

$$
\frac{d m_{R}}{d t}=-\frac{d m_{P}}{d t}
$$

Although general for closed systems, this relation does not allow us to relate variations in the mass of a specific substance during a reaction with changes in the mass of any other. In other words, you cannot derive Eq. 13 from the relations just presented:

$$
\frac{\mathrm{dm}_{\mathrm{i}}}{\mathrm{dt}}=-\frac{\mathrm{dm_{j }}}{\mathrm{dt}} \quad \text { (uncorrect) }
$$

for any reagent $i$ or product $j$, with masses $m_{i}$ and $m_{j}$, respectively. Its not possible to prove $d m_{i} / d t=$ $\mathrm{K}(i, j) \mathrm{dm}_{\mathrm{j}} / \mathrm{dt}$ for a proportionality constant $\mathrm{K}(i, j)$ (which depends on both $i$ and $j$ ) either, because no information with regard to the stoichiometry of the reaction and the relation between mass and amounts of substances have been provided so far.

One can demonstrate, however, that (from Eq. 11):

$$
\begin{gathered}
\frac{d m_{R}}{d t}=\frac{d}{d t} \sum_{i} m_{i}=-\frac{d m_{P}}{d t}=-\frac{d}{d t} \sum_{j} m_{j} \\
\sum_{i} \frac{d m_{i}}{d t}=-\sum_{j} \frac{d m_{j}}{d t}
\end{gathered}
$$


Naturally that Eq. 14 does not lead to Eq. 13 if considered alone. It says, though, that the sum of the variations on reagents masses through time is the negative of the sum of variations on products through the same time interval, despite the fact that individual variations of mass in certain reagent $i$ is not necessarily equivalent to the decrease in mass of any particular product. The mass conservation is a global concept, not a molecular one (at least not directly).

We can represent mass conservation as the sum of the mass changes being equal to zero for any $k$, either reagent or product:

$$
\sum_{k} \frac{d m_{k}}{d t}=\sum_{i} \frac{d m_{i}}{d t}+\sum_{j} \frac{d m_{j}}{d t}=0
$$

Which is sometimes abbreviated as $\sum_{k} d m_{k}=0$. Therefore, the total mass $\sum_{k} m_{k}$ is conserved over time because it is not a function of $t$, implying its derivative with relation to time is a constant; in this case, zero.

Now we proceed to investigate reaction extent conservation. From Eq. 7:

$$
\frac{\mathrm{d} \xi / \mathrm{dt}}{\mathrm{d} n_{\mathrm{k}} / \mathrm{dt}}=\frac{\mathrm{d} \xi}{\mathrm{dn} n_{\mathrm{k}}}=\frac{1}{v_{\mathrm{k}}}(\forall \mathrm{k})
$$

Therefore, the changes in the reaction extent relative to the amount of any substance $k$ is constant for a specific amount of time, $v_{\mathrm{k}}^{-1}$, and if we sum the derivatives of all substances:

$$
\sum_{k} \frac{d \xi}{d n_{k}}=\sum_{k} \frac{1}{v_{k}}(\forall k)
$$

Here we have an analogous expression for mass conservation through time, $\sum_{\mathrm{k}}\left(\mathrm{dm} \mathrm{m}_{\mathrm{k}} / \mathrm{dt}\right)=0$ (the summation of mass variations through time is constant, and zero), but for the reaction extent conservation through changes in substance quantities, $\sum_{\mathrm{k}}\left(\mathrm{d} \xi / \mathrm{d} n_{\mathrm{k}}\right)=\sum_{\mathrm{k}} v_{\mathrm{k}}^{-1}$ (the summation in reaction extent changes relative to $\mathrm{n}$ over all substances is constant). The summation in Eq. 17 is not zero, but is constant over chemical transformations during certain amount of time. It is conserved through changes in quantities of any substance upon reaction in this time interval.

An important observation is that the reaction extent is defined under the assumption that there is no change in the chemical reaction followed during time: the stoichiometry of the reaction is not changed. This is clearly expressed by Eq. 17, where reaction extent is conserved only if the stoichiometric coefficients do not change. If more than one reaction with distinct stoichiometry is present, then more than one reaction extent must be defined [7].

\section{Mass conservation in chemical reactions}

The usual expression for mass conservation in chemical reactions is based on the sum of stoichiometric numbers of the substances $(v)$ multiplied by the respective molar masses $(M)[3,11,12]$ :

$$
\sum_{k} v_{k} M_{k}=0
$$

Eq. 18 is the mathematical expression implied when the chemical equation $\sum_{k} v_{k} A_{k}=0$, for chemical species $A_{k}$, is cited. It can be proved that Eq. 18 is a necessary and sufficient condition for Eq. 15 (which means that each one can be proved if one assumes the other):

$$
\left(\sum_{\mathrm{k}} \mathrm{d} \mathrm{m}_{\mathrm{k}}=0\right) \Leftrightarrow\left(\sum_{\mathrm{k}} v_{\mathrm{k}} \mathrm{M}_{\mathrm{k}}=0\right)
$$

It can be proved by direct use of the law of definite proportions [2], but we will show it by applying mass conservation in reaction extent definition.

Since the quantity of substance is just the mass over the molar mass, then for any pair of species $x$ and $y$ in Eq. 8:

$$
\frac{1}{v_{x} M_{x}} \frac{d m_{x}}{d t}=\frac{1}{v_{y} M_{y}} \frac{d m_{y}}{d t}(\forall x, y)
$$

Which leads to the correct form of 13 , relating the mass variations of any reagent $x=i$ with changes in a product $y=j$. The negative sign of Eq. 13 also appears in Eq. 20, but is canceled out because of the stoichiometric number definition (negative for reagents). If we sum up the reaction extents over all species:

$$
\begin{gathered}
\frac{1}{v_{k} M_{k}} \frac{d m_{k}}{d t}=\frac{d \xi}{d t}(\forall k) \\
\frac{1}{v_{k} M_{k}} \frac{d m_{k}}{d t}-\frac{d \xi}{d t}=0(\forall k) \\
\frac{1}{v_{k} M_{k}}\left(\frac{d m_{k}}{d t}-v_{k} M_{k} \frac{d \xi}{d t}\right)=0(\forall k) \\
\frac{d m_{k}}{d t}-v_{k} M_{k} \frac{d \xi}{d t}=0(\forall k) \\
\sum_{k} \frac{d m_{k}}{d t}-\frac{d \xi}{d t} \sum_{k} v_{k} M_{k}=0 \\
\sum_{k} \frac{d m_{k}}{d t}=\frac{d \xi}{d t} \sum_{k} v_{k} M_{k}
\end{gathered}
$$

If mass is conserved then $\sum_{k}\left(\mathrm{dm}_{\mathrm{k}} / \mathrm{dt}\right)$ must be zero. That implies $\sum_{k} v_{k} M_{k}=0$, since $d \xi / d t \neq 0$ (otherwise no reaction has occurred), which is the usual mathematical representation for mass conservation in chemical reactions. On the other hand, assuming $\sum_{k} v_{k} M_{k}=0$ leads directly to $\sum_{k} d m_{k} / d t=0$. Consequently, mass conservation is a necessary and sufficient condition for the quantity $\sum_{k} v_{k} M_{k}$ to conserve as well. 


\section{Conclusions}

Reaction extent is the way of counting how many reactions of some kind occurred in the system after some variation (usually through time) by the changes in the amount of any species involved. That is, for a given reaction, you can tell how many times the prototype of reaction you write, such as $3 \mathrm{~A} \longrightarrow 2 \mathrm{~B}$, had occurred after some time. This quantity can be defined, as usual in books, or proved to be a consequence of law of definite proportions in closed systems, an older (often forgotten) approach. We have shown an alternative version of this proof, which grounds the comprehension of the meaning of the reaction extent, and its relation with mass conservation, for the student to proceed in the advanced courses of Chemistry. Reaction extent conserves in closed systems, and this also is evidenced, in a distinct manner as mass conserves. The first conservation is a consequence of the closeness of the system; the second comes from the assumption that the reaction has specific and unchanged stoichiometric coefficients.

\section{Acknowledgments}

The author thanks prof. Janaina Versiani for the revision of the manuscript, and $\mathrm{CNPq}$ and Capes for the scholarship during the time this work has been done.

\section{References}

[1] Théophile De Donder. Leçons de Thermodynamique et de Chimie Physique. Gauthier-Villars, Paris, 1920.

[2] A. Dumon, A. Lichanot, and E. Poquet. Describing Chemical Transformations: From the Extent of Reaction $\xi$ to the Reaction Advancement Ratio $\chi$. Journal of Chemical Education, 70(1):29, 1993.

[3] Joseph Kestin. A Course in Thermodynamics, volume 2. Hemisphere Publishing Corporation, New York, 1979.

[4] Juan José Baeza-Baeza and María Celia GarcíaAlvarez-Coque. Extent of Reaction Balances: A Convenient Tool to Study Chemical Equilibria. World Journal of Chemical Education, 2(4):54-58, 2014.

[5] L. Arnaut, S. Formosinho, and H. Burrows. Chemical Kinetics From Molecular Structure to Chemical Reactivity. Elsevier, UK, 2007.

[6] Guy Schmitz. What Is a Reaction Rate? Journal of Chemical Education, 82(7):1091, 2005.

[7] Ilya Prigogine. Introduction to Thermodynamics of Irreversible Processes. Interscience Publishers, New York, 1961.

${ }^{[8]}$ D. Kondepudi and I. Prigogine. Modern Thermodynamics: from heat engines to dissipative structures. John Wiley \& Sons, UK, 2015.
[9] John F. Garst. The extent of reaction as a unifying basis for stoichiometry in elementary chemistry. Journal of Chemical Education, 51(3):194, 1974.

[10] G. Moretti. The "extent of reaction": a powerful concept to study chemical transformations at the first-year general chemistry courses. Foundations of Chemistry, 17(2):107-115, 2015.

[11] T. De Donder and P. V. Rysselberghe. Thermodynamic Theory of Affinity: A book of principles. Standford University Press, California, 1936.

${ }^{[12]}$ I. Prigogine, R. Defay, and D. E. Everett. Chemical Thermodynamics. Longmans Green and Co, London, 1954.

[13] N. C. Craig. The Chemist's Delta. Journal of Chemical Education, 64(8):668-669, 1987.

[14] Michael C. Sostarecz and Audra Goach Sostarecz. A Conceptual Approach to Limiting-Reagent Problems. Journal of Chemical Education, 89:1148-1151, 2012.

[15] J. E. Vandezande, D. A. V., and R. L. DeKock. Reaction Extrema: Extent of Reaction in General Chemistry. Journal of Chemical Education, 90(9):1177-1179, 2013. 